\title{
Combined Antioxidant, Anti-inflammaging and Mesenchymal Stem Cell Treatment: A Possible Therapeutic Direction in Elderly Patients with Chronic Obstructive Pulmonary Disease
}

\author{
Shijin Xia ${ }^{1, \#, ~ C h a n g x i ~ Z h o u ~}{ }^{2, \#}$, Bill Kalionis ${ }^{3}$, Xiaoping Shuang ${ }^{4}$, Haiyan Ge ${ }^{5^{*}}$, Wen Gao ${ }^{* *}$ \\ ${ }^{1}$ Shanghai Institute of Geriatrics, Huadong Hospital, Fudan University, Shanghai, China. \\ ${ }^{2}$ Department of Respiratory Medicine, The Second Medical Center of PLA General Hospital, Beijing, China. \\ ${ }^{3}$ Department of Maternal-Fetal Medicine Pregnancy Research Centre and University of Melbourne Department \\ of Obstetrics and Gynaecology, Royal Women's Hospital, Parkville, Victoria, Australia. \\ ${ }^{4}$ Department of Cardiovascular Diseases, Xiangyang Hospital of Traditional Chinese Medicine, Xiangyang, \\ Hubei, China. \\ ${ }^{5}$ Department of Pulmonary Diseases, Huadong Hospital, Fudan University, Shanghai, China. \\ ${ }^{6}$ Department of Thoracic Surgery, Huadong Hospital, Fudan University, Shanghai, China.
}

[Received February 28, 2019; Revised April 21, 2019; Accepted May 8, 2019]

\begin{abstract}
Chronic Obstructive Pulmonary Disease (COPD) is a worldwide health problem associated with high morbidity and mortality, especially in elderly patients. Aging functions include mitochondrial dysfunction, cell-to-cell information exchange, protein homeostasis and extracellular matrix dysregulation, which are closely related to chronic inflammatory response and oxidation-antioxidant imbalance in the pathogenesis of COPD. COPD displays distinct inflammaging features, including increased cellular senescence and oxidative stress, stem cell exhaustion, alterations in the extracellular matrix, reduced levels of endogenous anti-inflammaging molecules, and reduced autophagy. Given that COPD and inflammaging share similar general features, it is very important to identify the specific mechanisms of inflammaging, which involve oxidative stress, inflammation and lung mesenchymal stem cell function in the development of COPD, especially in elderly COPD patients. In this review, we highlight the studies relevant to COPD progression, and focus on mechanisms associated with inflammaging.
\end{abstract}

Key words: chronic obstructive pulmonary disease, oxidative stress, inflammaging, lung mesenchymal stem cells

\section{Overview of aging and COPD}

Chronic Obstructive Pulmonary Disease (COPD) is a common public health problem associated with high morbidity and mortality. Worldwide, the Global Burden of Disease study in 2015 estimated that 174.5 million adults had prevalent COPD [1], and spirometry-defined COPD suggested as many as 384 million adults were affected [2]. The China Pulmonary Health $(\mathrm{CPH})$ study revealed the spirometry-defined overall prevalence of COPD was $8.6 \%$, which accounted for 99.9 million Chinese adults aged 20 years or older [3]. Globally,

*Correspondence should be addressed to: Drs Haiyan Ge and Wen Gao, Huadong Hospital, Fudan University, Shanghai, China. Email: haiyange@hotmail.com and gaowen5921@163.com. \#These authors contributed equally to this work.

Copyright: () 2019 Xia S et al. This is an open-access article distributed under the terms of the Creative Commons Attribution License, which permits unrestricted use, distribution, and reproduction in any medium, provided the original author and source are credited. 
COPD is a major burden to current societies and economies. By 2030, COPD is expected to be the fourth leading cause of death $[4,5]$.

With regard to aging of the population, the $\mathrm{CPH}$ study showed that among people aged 40 years or more, the incidence of COPD was much higher, at $13.7 \%$ [3]. COPD surveillance in United States showed the prevalence of COPD increased with advancing age at $6.6 \%$ for those aged $45-54$ years, $9.2 \%$ for those aged 5564 years, and $12.1 \%$ for those aged 65-74 years [6]. Similarly, the mortality attributable to COPD increased with age [7]. Impaired tissue growth and organ dysfunction are characteristics of aging, and aging is the greatest risk factor for chronic, non-communicable diseases $[8,9]$. During aging, chronic, sterile, low-grade inflammation, called inflammaging, develops and this contributes to the pathogenesis of COPD [10]. Current treatment measures for COPD such as bronchiectasis, anti-inflammaging drugs and anti-oxidants only relieve symptoms, or reduce the occurrence of acute exacerbations, but they do not delay or prevent the progression of the disease [11]. To date, there are no effective countermeasures to the deterioration of lung function and disease progression in elderly COPD patients.

Aging functions include mitochondrial dysfunction, cell-to-cell information exchange, protein homeostasis and extracellular matrix dysregulation, all of which are also closely associated with chronic inflammatory response and oxidant-antioxidant imbalance in the pathogenesis of COPD. COPD displays other characteristics of aging such as stem cell exhaustion, oxidative stress, cellular senescence, abnormal extracellular matrix and a reduction in endogenous antiaging molecules. Recent studies show oxidative stress accelerates aging, and a consequence is that stem cell populations are depleted, antioxidant defenses are reduced, and there is defective mitochondrial function, all of which generate additional oxidative stress [12].

Since COPD and inflammaging share similar characteristics, it is imperative for a better understanding of the development of COPD to identify and elucidate the mechanisms of oxidative stress, inflammaging and lung mesenchymal stem cell function. This is especially important in elderly COPD patients. In this review, we conducted an extensive literature appraisal of inflammaging-related studies associated with COPD. The aim was to assess our knowledge of the specific mechanisms operating in COPD that are responsible for oxidative stress, inflammaging and abnormal lung mesenchymal stem cell function, and their interactions. In addition, we used this knowledge to explore potential new treatment strategies aimed at delaying or preventing the progression of COPD.

\section{Oxidative stress and COPD}

\subsection{Oxidation-antioxidant imbalance is involved in the development of COPD}

Several mechanisms associated with aging including oxidative stress, shortened telomere length and cellular senescence, are potentially involved in the pathogenesis of COPD [13]. Previous studies showed that increased levels of biomarkers of oxidative stress (8oxodG, NT, F2IsoPs and AGEs) were strongly correlated with the severity of airflow limitation in COPD elderly patients [14]. Decreased levels of sRAGE and esRAGE have been detected in COPD elderly patients (i.e. with a mean age of 63 years), and their reduced levels have a significant association with forced expiratory volume in 1 second (FEV1) and FEV1/ forced vital capacity (FVC), and age demonstrated a covariation of sRAGE [15]. Oxidantantioxidant imbalance plays a crucial role in the development of COPD. Oxidative stress caused by smoking and environmental pollution can lead to extensive tissue damage and COPD exacerbation in patients at about 63 years of age $[16,17]$. Some markers, such as MDA in sputum, appear to be useful for monitoring exacerbation-associated oxidative stress in COPD [18]. Oxidative stress is a consequence of the action of reactive oxygen species (ROS). Exacerbation of COPD patients showed markedly increased ROS production in sputum neutrophils [19]. ROS are mainly comprised of the superoxide radical $\mathrm{O}_{2}^{-}$and hydrogen peroxide $\left(\mathrm{H}_{2} \mathrm{O}_{2}\right)$. ROS molecules such as $\mathrm{O}_{2}^{-}$, ONOO', $\mathrm{H}_{2} \mathrm{O}_{2}$, and $\mathrm{OH}^{-}$cause damage to the integrity of airway epithelial cells. Nrf2 (nuclear factor-E2-related factor 2) is the major transcription factor that controls antioxidant responses. The expression level of Nrf2 decreases in the chronic obstructive lung, thereby unbalancing the oxidant-antioxidant levels. [20]. Activation of NADPH oxidase 2 (Nox2) in neutrophils, macrophages and epithelial cells may produce the superoxide radical $\mathrm{O}_{2}$ $\mathrm{O}_{2}{ }^{-}$either forms a peroxynitrite ONOO- with nitric oxide (NO) or rapidly forms hydrogen peroxide $\left(\mathrm{H}_{2} \mathrm{O}_{2}\right)$ under the action of superoxide dismutase (SOD). Under normal conditions, $\mathrm{H}_{2} \mathrm{O}_{2}$ is metabolized into water and oxygen by glutathione peroxidase (Gpxs), catalase (Cat) and Prdx6. However, in elderly patients with COPD, the levels of Gpxs and Cat decrease severely, which leads to a further increase of $\mathrm{H}_{2} \mathrm{O}_{2}$.

Previous studies found that the plasma oxidative stress marker lipid peroxide MDA (malondialdehyde) was significantly elevated in patients with COPD. The MDA level in patients with severe COPD was significantly higher than in patients with mild to moderate COPD. There is a negative correlation between MDA level and forced expiratory volume in one second (FEV1)\% $(P<$ 
0.001) [21, 22]. The concentration of $\mathrm{H}_{2} \mathrm{O}_{2}$ in the exhaled breath of patients with smoking-induced COPD was significantly higher than in patients with non-smoking COPD. $\mathrm{H}_{2} \mathrm{O}_{2}$ was further elevated when the condition worsened. $\mathrm{H}_{2} \mathrm{O}_{2}$ levels are elevated in elderly patients with COPD due to various aging factors, which exacerbates oxidative stress.

The above data indicate that the oxidant-antioxidant imbalance is manifested by abnormal oxidative free radical scavenging, and it induces DNA damage and premature senescence [23]. This imbalance is a key factor involved in the development and progression of COPD, which is more severe in elderly patients with COPD.

\subsection{Abnormalities in CFTR/pendrin transport and redox products of ion channel regulation, are involved in oxidative stress imbalance in COPD}

CFTR (cystic fibrosis transmembrane conductance regulator) is the main ion channel for the secretion of fluid from the airway epithelial cells. There are two NBD (nucleotide binding site) active sites in the cytoplasm. Phosphorylation of NBD1 in combination with ATP initiates the opening of the CFTR channel, whereas ATP dephosphorylation into ADP, combined with NBD-2, closes the CFTR channel [24]. After activation of CFTR, the channel is open, and chloride ions are transported from inside to the outside of cell in order to maintain the stability of the airway surface liquid (ASL) [25]. In addition to transporting chloride ions, CFTR transports glutathione (GSH). GSH has a reducing function, which maintains a relatively high reduction state of ASL. GSH reduces the cross-linking reaction of mucin, and reduces the production of free radicals, leading to an antioxidative stress and anti-inflammaging state. A recent study showed that COPD emphysema pathogenesis is alleviated by treatment with a potent anti-oxidant with CFTR/autophagy-augmenting properties [26]. Another study showed that reduced $\beta$-adrenergic sweat rate, which reflecting acquired CFTR dysfunction and sweat chloride are associated with COPD severity and clinical symptoms, and univariate analysis revealed a significant relationship with age [27]. The CFTR M470V gene variant may be a potential modifier of COPD severity [28]. Our studies and related studies found that CFTR down-regulation or inhibition causes the following changes: (1) ASL thickness is reduced by $30 \%$; (2) ASL viscosity is increased 5 fold if accompanied by upregulation of epithelial cell $\mathrm{Na}^{+}$channel $\mathrm{ENaC}$ function; (3) CFTR inhibits inflammation, and the ability of epithelial cells to release inflammatory factors is enhanced after CFTR inhibition; (4) CFTR affects cell migration and post-injury repair; (5) since CFTR can transport GSH, inhibition of CFTR increases oxidative stress response of epithelial cells and the removal of sulfhydryl groups on mucins, and increases the viscosity of cross-linked aggravated mucus [29-32].

The studies described above suggest that CFTR plays an important role in chronic inflammation of the airways and in balancing oxidative/antioxidants. If CFTR is down regulated or inhibited, it affects airway mucus clearance and aggravates both the inflammatory response and epithelial oxidative stress. Studies show that CFTR on the cell membrane is significantly decreased in epithelial cells exposed to cigarette extracts, resulting in decreased thickness of the air layer of the respiratory epithelium [33]. In vitro, CFTR, MRP2, or BCRP inhibition decreases GSH efflux after exposure to cigarette smoke extract. In a murine model, CFTR-, BCRP-, or MRP2deficient mice were exposed to either air or acute CS. Only CFTR-deficient mice had reduced basal and CSinduced GSH in the epithelial lining fluid (ELF). BCRPor MRP2-deficiency showed no effect on ELF, GSH basal or CS-exposed levels [34]. Reduced CFTR expression decreases the transport of GSH, increases the viscosity of secretions, and leads to decreased respiratory dysfunction and decreased antioxidant capacity. Therefore, upregulating CFTR channel function or increasing its cell membrane expression may be a novel strategy for intervention in COPD.

Pendrin (SLC26A4) is an important member of SLC26 family of proteins, is expressed on the surface of airway epithelial cells, and participates in $\mathrm{Cl}^{-} / \mathrm{HCO}^{-}$ transportation. Pendrin is mainly involved in thickness regulation of ASL, and the expression of mucin. In addition to the above effects, pendrin transports thiocyanate $\left(\mathrm{SNC}^{-}\right)$to $\mathrm{ASL}$ and participates in the production of the antibacterial molecule $\mathrm{OSCN}^{-}$[35]. CFTR interacts with pendrin, and the $\mathrm{R}$ region of CFTR is phosphorylated by PKA to bind to pendrin's STAS (sulfate transporter and anti-sigma factor antagonist domain), which activates pendrin [36-38]. A recent study showed that pendrin mediates bicarbonate secretion and enhances CFTR function in airway surface epithelia [39]. Whether pendrin is synergistic with CFTR transport of GSH is still unclear. Further studies should focus on employing inhibitors and activators of CFTR, inhibitors of pendrin, and highly specific small molecule compounds obtained by high-throughput screening, to regulate the function of these channels and provide important information for improving respiratory tract function (Fig. 1).

To summarize, oxidative stress adversely affects the microenvironment of respiratory epithelial cells and impairs epithelial integrity. CFTR maintains a stable and reduced oxidative stress state in the respiratory epithelial microenvironment, participates in the migration and repair of respiratory epithelial cells and contributes to 
anti-inflammaging and anti-oxidative responses. CFTR interacts with pendrin to maintain the stability of the respiratory epithelial microenvironment. Manipulating the expression of CFTR/pendrin in epithelial cells, developing effective channel inhibitors and activators to regulate anti-oxidative function and efficacy are strategies that have potential clinical significance in treating elderly patients with COPD.

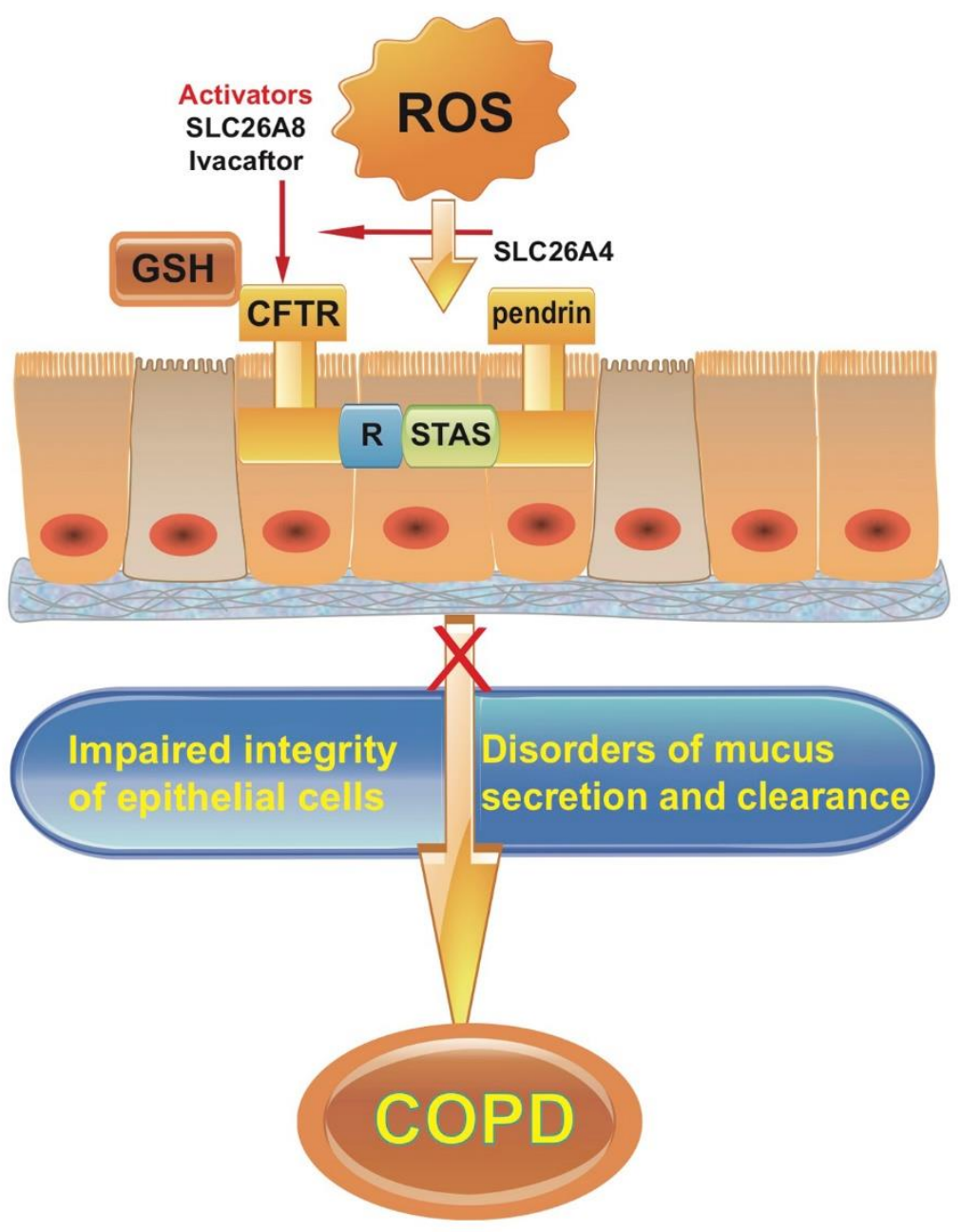

Figure 1. Oxidative stress interacts with airway epithelial cells to participate in the development of COPD. Activators of CFTR, such as pendrin (SLC26A4), SLC26A8 and Ivacaftor, may improve respiratory tract function and delay the process of COPD.

\section{Inflammaging and COPD}

\subsection{Inflammaging is the key cause of lung damage and the development COPD in elderly patients}

The main features of inflammaging are chronic progressive elevation of the pro-inflammatory state and age-related chronic inflammatory processes, which are low, uncontrollable, asymptomatic, chronic, and systemic. Our research and that of others suggests that inflammaging is closely related to geriatric diseases and is the underlying cause of COPD [40-42]. Inflammaging is a key link in the conversion of inflammation to COPD [43-45]. A study in a population aged about 70 years showed that the decrease in growth differentiation factor 11 (GDF11), an anti-ageing factor, may be involved in the cellular senescence observed in COPD [46].

The decline and onset of COPD in the elderly is closely related to systemic low-grade chronic inflammation, the so-called inflamm-ageing (also called inflammaging) hypothesis, and this might add to the burden of COPD in the elderly [47]. A meta-analysis 
showed that the pathogenesis of COPD is closely related to the increase of serum leukocytes, IL-6, IL-8, C-reactive protein (CRP) and fibrinogen [48]. Cell senescence is an important factor in the induction of chronic obstructive pulmonary inflammation. Aging eventually leads to the recruitment and colonization of lung neutrophils, macrophages and $\mathrm{T}$ cells in patients with COPD caused by smoking. Once inflammation is triggered, it causes a series of permanent inflammations and damages the lung parenchyma, which eventually results in the development of COPD. Therefore, intervention in aging may be an important breakthrough in the prevention, delaying or treatment of lung disease. Most important is that the elderly population can present with diminished lung function but without the presence of clinical COPD. Therefore, a confident clinical diagnosis of COPD needs to be made [49].

There are many theories about the mechanism of inflammaging. The theory of oxidation-inflammaging and the theory of stem cell aging are particularly important. Based on the close association between oxidative stress, inflammation, and aging, De la Fuente $M$ et al. [50] proposed the oxidation-inflammation theory of aging. The theory holds that oxidative stress leads to inflammaging. Glucocorticoid resistance, sympathetic nervous system function changes, and parasympathetic nervous system function changes during chronic stress may be the mechanism of stress-induced inflammation [51, 52]. Inflammaging is closely related to stem cell aging. Chronic inflammation induces stem cell senescence during pathological processes of inflammaging. Studies show that mutation of the WRN gene in human stem cells produces features of premature aging, which include slowing of growth, increased DNA damage response, and secretion of a large number of inflammatory factors. Moreover, these mutant stem cells also exhibit accelerated loss of heterochromatin, which decreases heterochromatin stability, and in turn induces cellular senescence [53]. Another study found that AU-rich-binding factor 1 (AUF1) controls both aging and inflammatory processes, not only by controlling inflammatory responses, but also by repairing telomerase to restore the length of telomeres at the ends of chromosomes and thereby prevent the acceleration of aging [54].

Clearly, it is very important to determine the connections and interactions between aging, inflammation, oxidative stress, stem cells, and COPD. We propose that a better understanding of the above pathophysiological mechanisms and stem cell functions is needed. This will provide the foundation for novel strategies to simultaneously control oxidative stress, inflammation, and aging, which will delay the aging process in lungs and thereby prevent and/or treat COPD.

\subsection{SIRT6 slows down the process of COPD by inhibiting the inflammaging pathway}

Sirtuin 6 (SIRT6) is a member of the sirtuin family of NAD-dependent enzymes and is one of the few genes that regulates longevity and aging. SIRT6 plays a role in DNA repair, telomerase function, genome stability and cell senescence [55]. In SIRT6-deficient mice, loss of the single-strand DNA damage repair function leads to genomic instability [56]. Our previous study reported that icariin (ICA) up-regulates SIRT6 protein expression, inhibits NF- $\mathrm{KB}(\mathrm{p} 65)$ protein expression and reduces the expression of downstream inflammatory cytokines in aged mice. ICA down-regulates target genes (i.e.TNF- $\alpha$, ICAM-1, IL-2, IL-6 and NF- $\kappa$ B) by acting directly or indirectly on SIRT6 [57]. Takasaka et al [58] found that SIRT6 expression was decreased in lungs of patients with COPD, whereas overexpression of SIRT6 inhibited smoking-induced senescence of human bronchial epithelial cells. Therefore, the properties of SIRT6 in delaying aging, and its anti-inflammaging action, make it a new target for the treatment of COPD. Further studies should explore the expression of SIRT6 in elderly patients with COPD, and the effect of SIRT6 on chronic obstructive pulmonary airway inflammation.

\subsection{SIRT6 controls the pathophysiological mechanism of COPD by regulating PAI-1}

Plasminogen activator inhibitor-1 (PAI-1) is expressed by inflammatory cells such as monocytes, neutrophils, mast cells, and activated T lymphocytes. PAI- 1 also affects the migration and activation of these inflammatory cells. PAI1 levels are significantly elevated in various senescent cells (e.g. fibroblasts and endothelial cells). Klotho (kl/kl) deficient mice are used as an aging model and show significantly higher levels of PAI-1 in plasma and tissues than normal mice [59]. Plasma PAI-1 concentrations are above the upper limit of normal in patients with the hereditary premature aging Werner syndrome [60]. Therefore, PAI-1, is considered to be a marker molecule for aging and is widely used in aging-related studies both in vitro and in vivo. PAI-1 affects inflammatory factor levels and cell migration processes. Studies show increased levels of PAI-1 in patients with COPD and this is associated with oxidative stress-induced activation of NF- $\kappa B$ [61]. A previous study reported that the concentration of PAI-1 in alveolar lavage fluid correlated with mortality in patients with compromised host defense mechanisms [62]. We used the mouse PAI-1 knockout model and found that PAI-1 is a key regulator of early lung inflammation, which affects the recruitment of neutrophils in the lung when knocked out [63]. The pivotal role of PAI-1 in the development of inflammation 
in the lungs, its mode of activation (which is dependent on the NF- $\kappa \mathrm{B}$ pathway), and particularly its negative effects on aging, suggest PAI-1 may be a downstream target of the SIRT6 pathway (Fig. 2).

Actively exploring the role and mechanism of action of inflammaging in COPD and using this information to delay the development of COPD through antiinflammaging and anti-aging treatments, is a new direction and new strategy for the prevention and treatment of COPD.

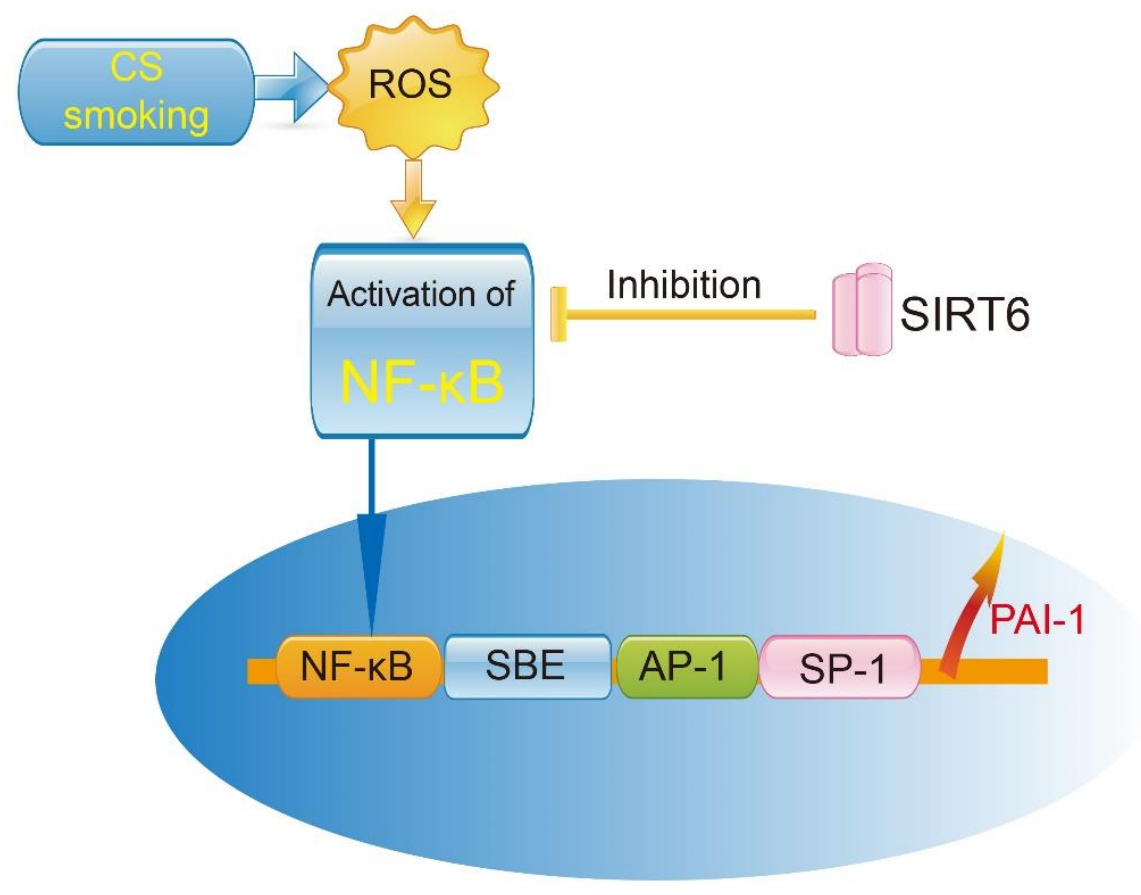

Figure 2. Effect of SIRT6 and PAI-1 on smoking-induced pulmonary inflammation.

\section{Inflammaging and oxidative stress affect mesenchymal stem cells during the development of COPD}

Mesenchymal stem cells have the ability to self-renew and have multipotent differentiation potential. These cells play an important role in tissue damage repair, homeostasis maintenance and immune regulation [64, 65]. In the lung, stem and progenitor cells are the main regenerative cells, which maintain the steady state and repair damage to lung epithelial cells. Current studies identify a variety of lung-derived stem/progenitor cells with self-renewal and differentiation potential, which include basal cells from the airway, secretory rod-like cells and alveolar type II epithelial cells from the alveoli, and mesenchymal stem cells from the pulmonary stroma [66]. Direct delivery of FGF-10 in the lungs of rats increases lung resident-MSCs in the treated lungs, which suggests that the protective effect of FGF-10 could be mediated, at least in part, by mobilizing lung residentMSCs [67]. MSC therapy is an important tool for regenerative medicine. Many studies have confirmed that MSCs have obvious therapeutic effects in various acute and chronic lung diseases [66]. A previous study showed that MSCs can treat lipopolysaccharide induced acute lung injury and ischemia-reperfusion induced lung injury [68].

\subsection{Application of MSC therapy for COPD}

In a COPD animal model, exogenous MSCs reduced the destruction of emphysema and pulmonary function caused by cigarette smoke [69, 70]. MSCs increased the expression of vascular endothelial growth factor (VEGF), VEGF receptors and TGF-beta 1, reduced lung cell apoptosis, inhibited the inflammatory cytokines TNF alpha, IL-1 beta, MCP-1, as well as reducing the secretion of IL-6 [69]. Clinical trials based on MSC therapy for COPD are underway, these trials use MSCs in different ways to obtain a therapeutic outcome. A placebocontrolled, randomized trial of MSCs in COPD in patients around 68 years old suggested there were no significant 
differences in pulmonary function tests or quality-of-life indicators following MSC administration. However an early, significant decrease in levels of circulating Creactive protein (CRP) was observed, and MSC administration appears to be safe in patients with moderate to severe COPD [71]. Another study using oneway endobronchial valves (EBV) together with MSC administration, provided evidence of decreased levels of circulating CRP, BODE (Body mass index, airway obstruction, dyspnea, and exercise index) and MMRC (Modified Medical Research Council) scores [72]. Systemic MSC infusion may be useful in the attenuation of inflammation in COPD patients [73]. Other studies verified that autologous MSC treatment in severe emphysema is feasible and safe [74].

From the above research, we propose that current clinical trials on patients with COPD support that MSC therapy improves inflammatory responses in patients with COPD, but the improvement of pulmonary function is not obvious [75]. Thus, the efficacy of MSCs in the treatment of COPD remains controversial. From the perspective of treatment, a one-time administration of MSCs can alleviate the inflammatory reactions in the airway and lung through a paracrine effect, but whether this permanently improves the airway reconstruction is more important and will most likely rely on the number and activity of endogenous MSCs in bodily tissues. COPD is a disease that accelerates aging of the lungs and is closely associated with other aging effects [76]. Aging leads to a decrease in the number and activity of stem cells in the trachea tissue and a consequence is a significant decrease in the ability to repair tissue [77]. Elderly patients with COPD have significantly reduced stem cell numbers and activity. Therefore, it is important to understand the effects of senescence, oxidative stress and chronic inflammation on both endogenous MSCs in the lungs of COPD patients, and on exogenous, administered MSCs used to treat COPD.

\subsection{Inflammaging and oxidative stress associated with COPD lead to an abnormal microenvironment for endogenous MSCs}

Studies have found that compared with young mice, the number of alveolar type I and type II epithelial cells in elderly mice is generally decreased, and the regeneration capacity of alveolar type II epithelial cells is weakened [78]. Senescence of alveolar type II epithelial cells was also found in lung tissues of patients with COPD [79]. Bone marrow MSCs from elderly patients show increased levels of intracellular reactive oxygen species, DNA methylation changes, telomere shortening and increased aging-related protein expression of the beta-galactose glucoside enzyme, all of which contribute to inhibiting the differentiation of MSCs [80]. Moreover, bone marrow MSCs showed reduced proliferation, migration and altered immune response [81]. Gene expression profile studies found aging human MSCs show aging-related phenotypes, and contain a variety of highly expressed factors that promote inflammation [82]. Extracellular matrix (ECM) also plays an important role in lung stem cell injury repair. In aging connective tissue, type I collagen is increased while type III collagen, and proteoglycan expression is decreased [80]. Matrix composition changes with age and this affects adhesion, proliferation and migration of stem cells in the lung. In patients with COPD, alveolar structure is often damaged, which leads to the failure of endogenous lung stem/progenitor cells to reconstruct the functional lung structure. The lungs of COPD patients are more exposed to tobacco smoke, airborne particulate matter and pollutants, which cause an oxidant-antioxidant imbalance in the lungs, resulting in increased ROS production in the extracellular matrix. ROS cause cell DNA damage, promote the release of inflammatory cytokines, inhibit cell proliferation, promote apoptosis, and decompose extracellular matrix [83], which hinder the repair by lung stem cells.

We propose that the combination of abnormal stem cells malfunctioning in the hostile microenvironment of the lungs in patients with COPD, particularly in the elderly patients with COPD, contributes to the destruction of lung tissue and the irreversible decline in lung function. Methods to improve the activity of endogenous MSCs or supplement/replace their activity by the use of exogenous stem cells are needed. Moreover, treatments that provide a more conducive microenvironment for enhanced stem cell repair of damaged lungs in elderly patients with COPD are also required.

Oxidative stress and chronic inflammation accelerate the progression of stem cell aging and COPD through the following mechanisms. In COPD patients, excessive ROS lead to an increase in senescent cells, and the senescenceassociated secretory phenotype (commonly referred to as SASP) further stimulates inflammation, alveolar structural destruction and endothelial dysfunction. ROS also leads to loss of stem cell self-renewal capacity and stem cell depletion [84]. ROS mediated phosphorylation of p53 via p38 MAPK, induces the expression of the cell cycle inhibitor $\mathrm{p} 21$, thereby inhibiting cell proliferation [85]. In human endothelial cell-derived MSCs, oxidative stress causes rapid phosphorylation of the adaptor protein 53BP1, activating DNA damage responses and causing irreversible arrest of the cell cycle in the G0/G1 phase. DNA damage response activates p53, which up-regulates $\mathrm{p} 21$ and thereby inhibits $\mathrm{pRb}$ protein, leading to senescence growth inhibition [86]. The use of glutathione synthetase inhibitors to induce increased expression of 
ROS in stem cells aggravates DNA damage and leads to increased expression of cell cycle inhibitors p16, p14 and p21 [87]. In addition, senescent cells up-regulate the expression of the cell cycle inhibitor p16, which plays an important regulatory role in oxidative stress-induced stem cell senescence and proliferation inhibition. Upregulation of p16 is also closely related to p53 expression and telomere shortening. Studies show ROS regulate the epigenetic process of stem cells, promote their DNA methylation and acetylation, thereby inhibiting stem cell proliferation and differentiation [88]. Elevated ROS inhibits the expression of the deacetylase SIRT1 during COPD and aging. Spontaneous alveolar cavity enlargement occurred in SIRT1+/- mice, and activation of FOXO3-related pathways by cigarette smoke further aggravates alveolar cavity enlargement and cell senescence [89]. Studies in a murine model, implicated the ROS-producing enzyme NOX2 in the pathogenesis of human emphysema. In mice, macrophage-specific NOX2 contributed to elastase-induced emphysema via SIRT1/MMP-9 pathways [90]. In patients with COPD, SIRT1 expression is decreased, which in turn promotes the release of the inflammatory factor IL-8. At present, oxidative stress and chronic inflammation have a wellestablished role in the development and progression of COPD, however simple antioxidant and/or antiinflammaging treatments cannot control or reverse the clinical symptoms of COPD, especially in the elderly. Lung tissue destruction and lung function decline in patients with lung obstruction. Improving the stem cell microenvironment and restoring the repair function of endogenous stem cells is crucial in the treatment of chronic obstructive pulmonary disease, especially in elderly patients with chronic obstructive pulmonary disease. Other recent studies demonstrated that KGF-2 repairs alveolar epithelium and vascular endothelium by promoting proliferation of lung MSCs [91].

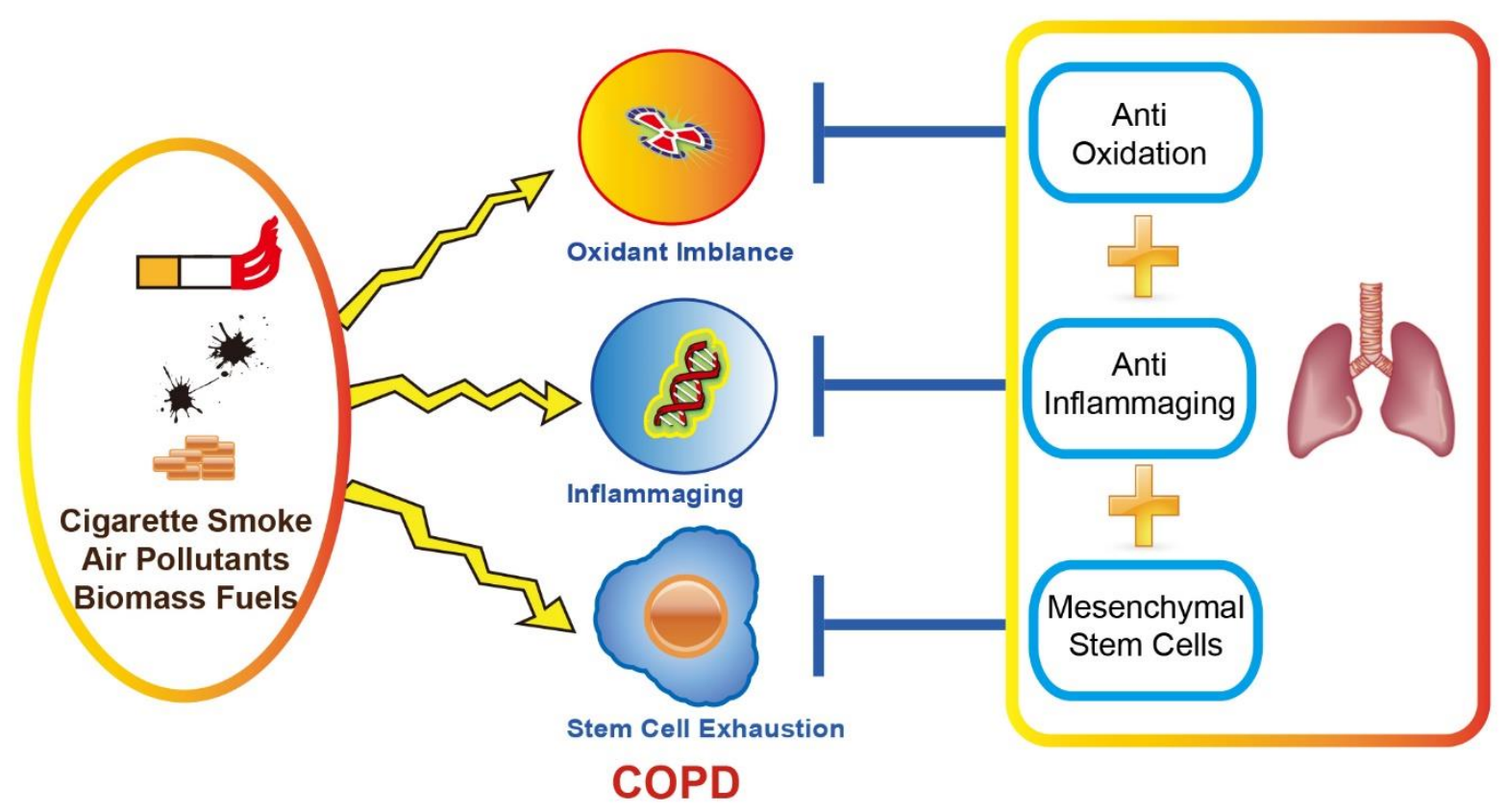

Figure 3. A new strategy for treatment of elderly patients with COPD; combining antioxidants and anti-inflammaging drugs with improved endogenous/exogenous MSC function.

\section{Possible therapeutic direction in elderly patients with COPD}

MSCs have the characteristics of multipotent differentiation potential, immune regulation and selfreplication. In addition to ROS-dependent depletion of MSCs, ROS also interacts with inflammatory aging, which causes an increase in senescent cell numbers and alters the microenvironment of MSCs in the lungs of
COPD. Based on the results of the studies described above, inflammaging, oxidative stress and stem cell dysfunction are involved in the development of COPD. Therefore, in addition to anti-inflammaging drugs and antioxidants, improving the microenvironment and repair function of MSCs is key to the treatment of chronic obstructive pulmonary disease (Figure 3 ). 
In this article, we reviewed the interaction between oxidative stress, inflammaging and stem cell dysfunction. On one hand, oxidative stress and inflammaging increases the aging-related phenotype, induces and aggravates the inflammatory response in the airway, and thus changes the microenvironment of stem cells with regard to their proliferation and differentiation. Moreover, oxidative stress and inflammaging leads to MSC exhaustion. Our research on COPD now focusses on the mechanism of oxidative stress and inflammaging, and the involvement of these mechanisms in MSC exhaustion. Employing a combination of antioxidants, anti-inflammatories and MSC treatment, is a possible therapeutic strategy for treating elderly patients with COPD.

\section{Acknowledgements}

This study was supported by the National Natural Science Foundation of China (81870044 and 81600056) and National Key R\&D Program of China (2018YFC2000 301).

\section{Conflict of interests}

The authors have no competing interests to declare.

\section{References}

[1] Mortality GBD, Causes of Death C (2016). Global, regional, and national life expectancy, all-cause mortality, and cause-specific mortality for 249 causes of death, 1980-2015: a systematic analysis for the Global Burden of Disease Study 2015. Lancet, 388: 1459-1544

[2] Adeloye D, Chua S, Lee C, Basquill C, Papana A, Theodoratou E, et al. (2015). Global and regional estimates of COPD prevalence: Systematic review and meta-analysis. J Glob Health, 5: 020415

[3] Wang C, Xu J, Yang L, Xu Y, Zhang X, Bai C, et al. (2018). Prevalence and risk factors of chronic obstructive pulmonary disease in China (the China Pulmonary Health [CPH] study): a national crosssectional study. Lancet, 391: 1706-1717

[4] Srivastava K, Thakur D, Sharma S, Punekar YS (2015). Systematic review of humanistic and economic burden of symptomatic chronic obstructive pulmonary disease. Pharmacoeconomics, 33: 467-488

[5] Sullivan SD, Ramsey SD, Lee TA (2000). The economic burden of COPD. Chest, 117: 5S-9S

[6] Ford ES, Croft JB, Mannino DM, Wheaton AG, Zhang X, Giles WH (2013). COPD surveillance--United States, 1999-2011. Chest, 144: 284-305

[7] Thannickal VJ, Murthy M, Balch WE, Chandel NS, Meiners S, Eickelberg O, et al. (2015). Blue journal conference. Aging and susceptibility to lung disease. Am J Respir Crit Care Med, 191: 261-269

[8] Lourenco J, Serrano A, Santos-Silva A, Gomes M,
Afonso C, Freitas P, et al. (2018). Cardiovascular Risk Factors Are Correlated with Low Cognitive Function among Older Adults Across Europe Based on The SHARE Database. Aging Dis, 9: 90-101

[9] Osanai T, Tanaka M, Mikami K, Kitajima M, Tomisawa T, Magota K, et al. (2018). Novel anti-aging gene NM 026333 contributes to proton-induced aging via NCX1-pathway. J Mol Cell Cardiol, 125: 174-184

[10] Franceschi C, Garagnani P, Parini P, Giuliani C, Santoro A (2018). Inflammaging: a new immunemetabolic viewpoint for age-related diseases. Nat Rev Endocrinol, 14: 576-590

[11] Bernardo I, Bozinovski S, Vlahos R (2015). Targeting oxidant-dependent mechanisms for the treatment of COPD and its comorbidities. Pharmacol Ther, 155: 6079

[12] Mercado N, Ito K, Barnes PJ (2015). Accelerated ageing of the lung in COPD: new concepts. Thorax, 70: 482-489

[13] Liguori I, Russo G, Curcio F, Bulli G, Aran L, DellaMorte D, et al. (2018). Oxidative stress, aging, and diseases. Clin Interv Aging, 13: 757-772

Choudhury G, MacNee W (2017). Role of Inflammation and Oxidative Stress in the Pathology of Ageing in COPD: Potential Therapeutic Interventions. COPD, 14: 122-135

[15] Gopal P, Reynaert NL, Scheijen JL, Schalkwijk CG, Franssen FM, Wouters EF, et al. (2014). Association of plasma sRAGE, but not esRAGE with lung function impairment in COPD. Respir Res, 15: 24

[16] Thomson NC (2018). Targeting oxidant-dependent mechanisms for the treatment of respiratory diseases and their comorbidities. Curr Opin Pharmacol, 40: 1-8

[17] Stanojkovic I, Kotur-Stevuljevic J, Milenkovic B, Spasic S, Vujic T, Stefanovic A, et al. (2011). Pulmonary function, oxidative stress and inflammatory markers in severe COPD exacerbation. Respir Med, 105 Suppl 1: S31-37

[18] Antus B, Harnasi G, Drozdovszky O, Barta I (2014). Monitoring oxidative stress during chronic obstructive pulmonary disease exacerbations using malondialdehyde. Respirology, 19: 74-79

[19] Vaitkus M, Lavinskiene S, Barkauskiene D, Bieksiene K, Jeroch J, Sakalauskas R (2013). Reactive oxygen species in peripheral blood and sputum neutrophils during bacterial and nonbacterial acute exacerbation of chronic obstructive pulmonary disease. Inflammation, 36: 1485-1493

[20] Dianat M, Radan M, Badavi M, Mard SA, Bayati V, Ahmadizadeh M (2018). Crocin attenuates cigarette smoke-induced lung injury and cardiac dysfunction by anti-oxidative effects: the role of $\mathrm{Nrf} 2$ antioxidant system in preventing oxidative stress. Respir Res, 19: 58

[21] Montano M, Cisneros J, Ramirez-Venegas A, PedrazaChaverri J, Mercado D, Ramos C, et al. (2010). Malondialdehyde and superoxide dismutase correlate with FEV(1) in patients with COPD associated with wood smoke exposure and tobacco smoking. Inhal Toxicol, 22: 868-874 
[22] To Y, Ito K, Kizawa Y, Failla M, Ito M, Kusama T, et al. (2010). Targeting phosphoinositide-3-kinase-delta with theophylline reverses corticosteroid insensitivity in chronic obstructive pulmonary disease. Am J Respir Crit Care Med, 182: 897-904

[23] Ahmad T, Sundar IK, Lerner CA, Gerloff J, Tormos AM, Yao H, et al. (2015). Impaired mitophagy leads to cigarette smoke stress-induced cellular senescence: implications for chronic obstructive pulmonary disease. FASEB J, 29: 2912-2929

[24] Sheppard DN, Welsh MJ (1999). Structure and function of the CFTR chloride channel. Physiol Rev, 79: $\mathrm{S} 23-45$

[25] Choi HC, Kim CS, Tarran R (2015). Automated acquisition and analysis of airway surface liquid height by confocal microscopy. Am J Physiol Lung Cell Mol Physiol, 309: L109-118

[26] Bodas M, Pehote G, Silverberg D, Gulbins E, Vij N (2019). Autophagy augmentation alleviates cigarette smoke-induced CFTR-dysfunction, ceramideaccumulation and COPD-emphysema pathogenesis. Free Radic Biol Med, 131: 81-97

[27] Courville CA, Tidwell S, Liu B, Accurso FJ, Dransfield MT, Rowe SM (2014). Acquired defects in CFTR-dependent beta-adrenergic sweat secretion in chronic obstructive pulmonary disease. Respir Res, 15: 25

[28] Stankovic M, Nikolic A, Divac A, Tomovic A, Petrovic-Stanojevic N, Andjelic M, et al. (2008). The CFTR M470V gene variant as a potential modifier of COPD severity: study of Serbian population. Genet Test, 12: 357-362

[29] Song Y, Namkung W, Nielson DW, Lee JW, Finkbeiner WE, Verkman AS (2009). Airway surface liquid depth measured in ex vivo fragments of pig and human trachea: dependence on $\mathrm{Na}+$ and $\mathrm{Cl}$ - channel function. Am J Physiol Lung Cell Mol Physiol, 297: L1131-1140

[30] Su X, Looney MR, Su HE, Lee JW, Song Y, Matthay MA (2011). Role of CFTR expressed by neutrophils in modulating acute lung inflammation and injury in mice. Inflamm Res, 60: 619-632

[31] Dalli J, Rosignoli G, Hayhoe RP, Edelman A, Perretti M (2010). CFTR inhibition provokes an inflammatory response associated with an imbalance of the annexin A1 pathway. Am J Pathol, 177: 176-186

[32] Sloane PA, Rowe SM (2010). Cystic fibrosis transmembrane conductance regulator protein repair as a therapeutic strategy in cystic fibrosis. Curr Opin Pulm Med, 16: 591-597

[33] Cantin AM, Hanrahan JW, Bilodeau G, Ellis L, Dupuis A, Liao J, et al. (2006). Cystic fibrosis transmembrane conductance regulator function is suppressed in cigarette smokers. Am J Respir Crit Care Med, 173: 1139-1144

[34] Gould NS, Min E, Martin RJ, Day BJ (2012). CFTR is the primary known apical glutathione transporter involved in cigarette smoke-induced adaptive responses in the lung. Free Radic Biol Med, 52: 12011206
[35]

Pedemonte N, Caci E, Sondo E, Caputo A, Rhoden K, Pfeffer U, et al. (2007). Thiocyanate transport in resting and IL-4-stimulated human bronchial epithelial cells: role of pendrin and anion channels. J Immunol, 178: 5144-5153

[36] Ko SB, Zeng W, Dorwart MR, Luo X, Kim KH, Millen L, et al. (2004). Gating of CFTR by the STAS domain of SLC26 transporters. Nat Cell Biol, 6: 343-350

[37] Shcheynikov N, Ko SB, Zeng W, Choi JY, Dorwart MR, Thomas PJ, et al. (2006). Regulatory interaction between CFTR and the SLC26 transporters. Novartis Found Symp, 273: 177-186; discussion 186-192, 261174

[38] Fong P (2012). CFTR-SLC26 transporter interactions in epithelia. Biophys Rev, 4: 107-116

[39] Kim D, Huang J, Billet A, Abu-Arish A, Goepp J, Matthes E, et al. (2019). Pendrin Mediates Bicarbonate Secretion and Enhances CFTR Function in Airway Surface Epithelia. Am J Respir Cell Mol Biol,

[40] Xia S, Zhang X, Zheng S, Khanabdali R, Kalionis B, $\mathrm{Wu}$ J, et al. (2016). An Update on Inflamm-Aging: Mechanisms, Prevention, and Treatment. J Immunol Res, 2016: 8426874

[41] Wu J, Xia S, Kalionis B, Wan W, Sun T (2014). The role of oxidative stress and inflammation in cardiovascular aging. Biomed Res Int, 2014: 615312 Franceschi C, Bonafe M, Valensin S, Olivieri F, De Luca M, Ottaviani E, et al. (2000). Inflamm-aging. An evolutionary perspective on immunosenescence. Ann N Y Acad Sci, 908: 244-254

[43] Franceschi C, Capri M, Monti D, Giunta S, Olivieri F, Sevini F, et al. (2007). Inflammaging and antiinflammaging: a systemic perspective on aging and longevity emerged from studies in humans. Mech Ageing Dev, 128: 92-105

[44] Kumar M, Seeger W, Voswinckel R (2014). Senescence-associated secretory phenotype and its possible role in chronic obstructive pulmonary disease. Am J Respir Cell Mol Biol, 51: 323-333

[45] Barnes PJ (2017). Senescence in COPD and Its Comorbidities. Annu Rev Physiol, 79: 517-539

[46] Onodera K, Sugiura H, Yamada M, Koarai A, Fujino N, Yanagisawa S, et al. (2017). Decrease in an antiageing factor, growth differentiation factor 11, in chronic obstructive pulmonary disease. Thorax, 72: 893-904

[47] De Martinis M, Franceschi C, Monti D, Ginaldi L (2005). Inflamm-ageing and lifelong antigenic load as major determinants of ageing rate and longevity. FEBS Lett, 579: 2035-2039

[48] Su B, Liu T, Fan H, Chen F, Ding H, Wu Z, et al. (2016). Inflammatory Markers and the Risk of Chronic Obstructive Pulmonary Disease: A Systematic Review and Meta-Analysis. PLoS One, 11: e 0150586

[49] Fragoso CA (2016). Epidemiology of Chronic Obstructive Pulmonary Disease (COPD) in Aging Populations. COPD, 13: 125-129

[50] De la Fuente M, Miquel J (2009). An update of the 
oxidation-inflammation theory of aging: the involvement of the immune system in oxi-inflammaging. Curr Pharm Des, 15: 3003-3026

[51] Almawi WY, Melemedjian OK (2002). Molecular mechanisms of glucocorticoid antiproliferative effects: antagonism of transcription factor activity by glucocorticoid receptor. J Leukoc Biol, 71: 9-15

[52] Miller GE, Cohen S, Ritchey AK (2002). Chronic psychological stress and the regulation of proinflammatory cytokines: a glucocorticoid-resistance model. Health Psychol, 21: 531-541

[53] Zhang W, Li J, Suzuki K, Qu J, Wang P, Zhou J, et al. (2015). Aging stem cells. A Werner syndrome stem cell model unveils heterochromatin alterations as a driver of human aging. Science, 348: 1160-1163

[54] Pont AR, Sadri N, Hsiao SJ, Smith S, Schneider RJ (2012). mRNA decay factor AUF1 maintains normal aging, telomere maintenance, and suppression of senescence by activation of telomerase transcription. Mol Cell, 47: 5-15

[55] Liao CY, Kennedy BK (2016). SIRT6, oxidative stress, and aging. Cell Res, 26: 143-144

[56] Mostoslavsky R, Chua KF, Lombard DB, Pang WW, Fischer MR, Gellon L, et al. (2006). Genomic instability and aging-like phenotype in the absence of mammalian SIRT6. Cell, 124: 315-329

[57] Chen Y, Sun T, Wu J, Kalionis B, Zhang C, Yuan D, et al. (2015). Icariin intervenes in cardiac inflammaging through upregulation of SIRT6 enzyme activity and inhibition of the NF-kappa B pathway. Biomed Res Int, 2015: 895976

[58] Takasaka N, Araya J, Hara H, Ito S, Kobayashi K, Kurita Y, et al. (2014). Autophagy induction by SIRT6 through attenuation of insulin-like growth factor signaling is involved in the regulation of human bronchial epithelial cell senescence. J Immunol, 192: 958-968

[59] Takeshita K, Yamamoto $\mathrm{K}$, Ito $\mathrm{M}$, Kondo $\mathrm{T}$, Matsushita T, Hirai M, et al. (2002). Increased expression of plasminogen activator inhibitor-1 with fibrin deposition in a murine model of aging, "Klotho" mouse. Semin Thromb Hemost, 28: 545-554

[60] Murano S, Nakazawa A, Saito I, Masuda M, Morisaki $\mathrm{N}$, Akikusa B, et al. (1997). Increased blood plasminogen activator inhibitor-1 and intercellular adhesion molecule-1 as possible risk factors of atherosclerosis in Werner syndrome. Gerontology, 43 Suppl 1: 43-52

[61] To M, Takagi D, Akashi K, Kano I, Haruki K, Barnes PJ, et al. (2013). Sputum plasminogen activator inhibitor-1 elevation by oxidative stress-dependent nuclear factor-kappaB activation in COPD. Chest, 144: 515-521

[62] Song Y, Lynch SV, Flanagan J, Zhuo H, Tom W, Dotson RH, et al. (2007). Increased plasminogen activator inhibitor-1 concentrations in bronchoalveolar lavage fluids are associated with increased mortality in a cohort of patients with Pseudomonas aeruginosa. Anesthesiology, 106: 252261
[63] Goolaerts A, Lafargue M, Song Y, Miyazawa B, Arjomandi M, Carles M, et al. (2011). PAI-1 is an essential component of the pulmonary host response during Pseudomonas aeruginosa pneumonia in mice. Thorax, 66: 788-796

[64] Rojas M, Xu J, Woods CR, Mora AL, Spears W, Roman J, et al. (2005). Bone marrow-derived mesenchymal stem cells in repair of the injured lung. Am J Respir Cell Mol Biol, 33: 145-152

[65] Ortiz LA, Dutreil M, Fattman C, Pandey AC, Torres G, Go K, et al. (2007). Interleukin 1 receptor antagonist mediates the antiinflammatory and antifibrotic effect of mesenchymal stem cells during lung injury. Proc Natl Acad Sci U S A, 104: 11002-11007

[66] Kotton DN, Morrisey EE (2014). Lung regeneration: mechanisms, applications and emerging stem cell populations. Nat Med, 20: 822-832

[67] Tong L, Zhou J, Rong L, Seeley EJ, Pan J, Zhu X, et al. (2016). Fibroblast Growth Factor-10 (FGF-10) Mobilizes Lung-resident Mesenchymal Stem Cells and Protects Against Acute Lung Injury. Sci Rep, 6: 21642

[68] Li J, Zhou J, Zhang D, Song Y, She J, Bai C (2015). Bone marrow-derived mesenchymal stem cells enhance autophagy via PI3K/AKT signalling to reduce the severity of ischaemia/reperfusion-induced lung injury. J Cell Mol Med, 19: 2341-2351

[69] Guan XJ, Song L, Han FF, Cui ZL, Chen X, Guo XJ, et al. (2013). Mesenchymal stem cells protect cigarette smoke-damaged lung and pulmonary function partly via VEGF-VEGF receptors. J Cell Biochem, 114: 323335

[70] Huh JW, Kim SY, Lee JH, Lee JS, Van Ta Q, Kim M, et al. (2011). Bone marrow cells repair cigarette smoke-induced emphysema in rats. Am J Physiol Lung Cell Mol Physiol, 301: L255-266

[71] Weiss DJ, Casaburi R, Flannery R, LeRoux-Williams M, Tashkin DP (2013). A placebo-controlled, randomized trial of mesenchymal stem cells in COPD. Chest, 143: 1590-1598

[72] de Oliveira HG, Cruz FF, Antunes MA, de Macedo Neto AV, Oliveira GA, Svartman FM, et al. (2017). Combined Bone Marrow-Derived Mesenchymal Stromal Cell Therapy and One-Way Endobronchial Valve Placement in Patients with Pulmonary Emphysema: A Phase I Clinical Trial. Stem Cells Transl Med, 6: 962-969

[73] Armitage J, Tan DBA, Troedson R, Young P, Lam KV, Shaw K, et al. (2018). Mesenchymal stromal cell infusion modulates systemic immunological responses in stable COPD patients: a phase I pilot study. Eur Respir J, 51

[74] Stolk J, Broekman W, Mauad T, Zwaginga JJ, Roelofs H, Fibbe WE, et al. (2016). A phase I study for intravenous autologous mesenchymal stromal cell administration to patients with severe emphysema. QJM, 109: 331-336

[75] Kubo H (2012). Concise review: clinical prospects for treating chronic obstructive pulmonary disease with regenerative approaches. Stem Cells Transl Med, 1: 
$627-631$

[76] Ito K, Barnes PJ (2009). COPD as a disease of accelerated lung aging. Chest, 135: 173-180

[77] Yin L, Zheng D, Limmon GV, Leung NH, Xu S, Rajapakse JC, et al. (2014). Aging exacerbates damage and delays repair of alveolar epithelia following influenza viral pneumonia. Respir Res, 15: 116

[78] Yang SR, Park JR, Kang KS (2015). Reactive Oxygen Species in Mesenchymal Stem Cell Aging: Implication to Lung Diseases. Oxid Med Cell Longev, 2015: 486263

[79] Tsuji T, Aoshiba K, Nagai A (2010). Alveolar cell senescence exacerbates pulmonary inflammation in patients with chronic obstructive pulmonary disease. Respiration, 80: 59-70

[80] Mora AL, Rojas M (2013). Adult stem cells for chronic lung diseases. Respirology, 18: 1041-1046

[81] Bustos ML, Huleihel L, Kapetanaki MG, LinoCardenas CL, Mroz L, Ellis BM, et al. (2014). Aging mesenchymal stem cells fail to protect because of impaired migration and antiinflammatory response. Am J Respir Crit Care Med, 189: 787-798

[82] Sepulveda JC, Tome M, Fernandez ME, Delgado M, Campisi J, Bernad A, et al. (2014). Cell senescence abrogates the therapeutic potential of human mesenchymal stem cells in the lethal endotoxemia model. Stem Cells, 32: 1865-1877

[83] Rahman I (2005). The role of oxidative stress in the pathogenesis of COPD: implications for therapy. Treat Respir Med, 4: 175-200

[84] Chilosi M, Carloni A, Rossi A, Poletti V (2013). Premature lung aging and cellular senescence in the pathogenesis of idiopathic pulmonary fibrosis and COPD/emphysema. Transl Res, 162: 156-173

[85] Borodkina A, Shatrova A, Abushik P, Nikolsky N, Burova E (2014). Interaction between ROS dependent DNA damage, mitochondria and p38 MAPK underlies senescence of human adult stem cells. Aging (Albany NY), 6: 481-495

[86] Chau BN, Wang JY (2003). Coordinated regulation of life and death by RB. Nat Rev Cancer, 3: 130-138

[87] Yahata T, Takanashi T, Muguruma Y, Ibrahim AA, Matsuzawa H, Uno T, et al. (2011). Accumulation of oxidative DNA damage restricts the self-renewal capacity of human hematopoietic stem cells. Blood, 118: $2941-2950$

[88] Sundar IK, Yao H, Rahman I (2013). Oxidative stress and chromatin remodeling in chronic obstructive pulmonary disease and smoking-related diseases. Antioxid Redox Signal, 18: 1956-1971

[89] Yao H, Chung S, Hwang JW, Rajendrasozhan S, Sundar IK, Dean DA, et al. (2012). SIRT1 protects against emphysema via FOXO3-mediated reduction of premature senescence in mice. J Clin Invest, 122: 2032-2045

[90] Trocme C, Deffert C, Cachat J, Donati Y, Tissot C, Papacatzis S, et al. (2015). Macrophage-specific NOX2 contributes to the development of lung emphysema through modulation of SIRT1/MMP-9 pathways. J Pathol, 235: 65-78

[91] Feng N, Wang Q, Zhou J, Li J, Wen X, Chen S, et al. (2016). Keratinocyte growth factor-2 inhibits bacterial infection with Pseudomonas aeruginosa pneumonia in a mouse model. J Infect Chemother, 22: 44-52 